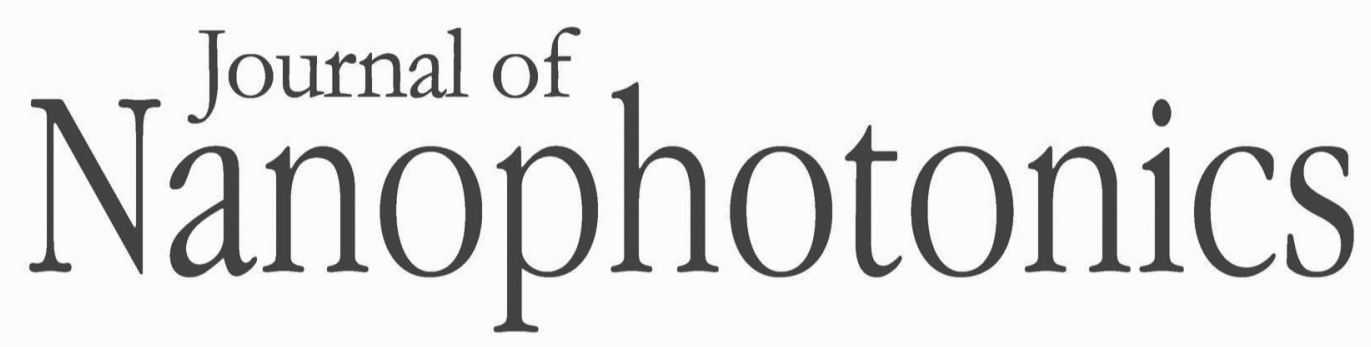

SPIEDigitalLibrary.org/jnp

\title{
Polymer-embedded colloidal lead- sulfide nanocrystals integrated to vertically slotted silicon-based ring resonators for telecom applications
}

Markus Humer

Romain Guider

Florian Hackl

Thomas Fromherz 


\title{
Polymer-embedded colloidal lead-sulfide nanocrystals integrated to vertically slotted silicon-based ring resonators for telecom applications
}

\author{
Markus Humer, Romain Guider, Florian Hackl, and Thomas Fromherz \\ Johannes Kepler University, Institute of Semiconductor and Solid State Physics, \\ Altenbergerstrasse 69, A-4040 Linz, Austria \\ markus.humer@jku.at
}

\begin{abstract}
The main drawback of the rapidly evolving field of silicon photonics lies in the absence of efficient monolithically integrated radiation sources as a consequence of the indirect bandgap of Si and Ge. Relevant alternatives based on the hybrid combination of Si with optically active materials have to be technologically simple, temporally stable, and provide efficient coupling to the Si waveguides. Lead-sulfide nanocrystals (NCs) were blended into a polymer resist suitable for deep-UV- and electron-beam lithography and integrated into Si-based vertically slotted waveguides and ring resonators. The polymer both stabilizes the NC's photoluminescence emission against degradation under ambient conditions and allows lithographic patterning of this compound material. After integration into the optoelectronic structures and upon optical pumping, intense photoluminescence emission from ring resonators was recorded at the output of bus-waveguides. The resonator quality factors were investigated for polymer-NC compounds with $\mathrm{NC}$ concentrations in the range between 0.1 and $8 \mathrm{vol} \%$. The spontaneous emission rate enhancement for vertically slotted resonators was estimated to be a factor of two higher as compared to unslotted ones. The stable integration of colloidal NCs as well as the improved light coupling to silicon circuits is an important step in the development of silicon-based hybrid photonics. () The Authors. Published by SPIE under a Creative Commons Attribution 3.0 Unported License. Distribution or reproduction of this work in whole or in part requires full attribution of the original publication, including its DOI. [DOI: 10.1117/1.JNP.7.073076]
\end{abstract}

Keywords: colloidal nanocrystals; lead sulfide; polymer; stability; ring resonator; photoluminescence; slot waveguide.

Paper 13077P received Sep. 4, 2013; revised manuscript received Nov. 4, 2013; accepted for publication Nov. 4, 2013; published online Nov. 27, 2013.

\section{Introduction}

The field of integrated Si-based photonics has been emerging as a promising alternative to electronics over the last years, a trend that can be explained by the rapid advancements in terms of device design and compatibility with complementary metal-oxide semiconductor technology. ${ }^{1}$ However, although many low-loss devices like waveguides, filters, and optical switches, have been demonstrated in the past, the indirect bandgap of Si makes the realization of integrated emitters a demanding task still left to be solved.

One approach to overcome this problem is the development of hybrid structures that introduce semiconductor nanocrystal quantum dots (NCs) as emitters into Si-based photonics. The absorption and emission wavelengths of these NCs depend on the materials used during synthesis and on their size, and especially lead-sulfide $(\mathrm{PbS}) \mathrm{NCs}$ are very attractive for applications in the telecom wavelength regime. ${ }^{2-4}$ Recent studies on NCs coupled to Si-based photonic crystal cavities have shown promising results toward the spectroscopy of single $\mathrm{NCs}^{5}$ as well as electrically pumped light-emitting devices. ${ }^{6}$

\footnotetext{
This paper is substantially based on the paper titled "Colloidal PbS nanocrystals integrated to Si-based photonics for applications at telecom wavelengths," presented at the SPIE Conference 8767 Integrated Photonics: Materials, Devices, and Applications II, held
} April 24-26, 2013, in Grenoble, Rhône-Alpes, France. 
As the fabrication of such NCs is based on colloidal chemistry, their on-chip integration can be carried out after synthesis using well-established methods like drop casting, spin coating, or inkjet printing. Alternatively, the NCs can be blended into suitable polymers, ${ }^{7,8}$ which protect the NCs from degradation caused by ambient conditions and allow lithographic methods for locally defined on-chip integration. While with respect to fabrication simplicity it can be very advantageous to implement the NCs on a fully completed optoelectronic chip, the question remains how the light emitted by the NCs can be efficiently coupled to the Si structures on the device.

In this work, we experimentally demonstrate the integration of polymer-embedded $\mathrm{PbS} \mathrm{NCs}$ into all-integrated Si-based vertically slotted ring resonators and waveguides as optically pumped emitters for room temperature applications. The novelty of this paper is in the significant increase of the spontaneous emission rate due to the vertical slot as compared to unslotted but otherwise similar structures that have been reported previously. ${ }^{9}$ Our method of integration allows the same device to be investigated as function of the NC concentration, using the negative electron-beam lithography (EBL) resist AR-7700.18 as polymer matrix. We demonstrate the full recovery of NC photoluminescence (PL) intensity after the polymer-NC blend is repeatedly heated up to $115^{\circ} \mathrm{C}$ and cooled back to room temperature, as required after exposure of the resist. Thus, spatial definition of a spin-cast polymer-NC film by EBL is possible without degrading the emission properties of the NCs. The compound additionally shows unaltered optical properties weeks after sample preparation, pointing out the high stability of this material and its suitability for device applications.

\section{Polymer Nanocrystal Compound}

As nanoemitters to be introduced into our photonic structures, colloidal PbS NCs from Evident Technologies (EviDot core LNR03LPB) suspended in toluene $(40 \mathrm{mg} / \mathrm{ml})$ with a core diameter of $\sim 5.1 \mathrm{~nm}$ are used. For an NC film drop cast onto a Si substrate, the room temperature PL spectrum under $532 \mathrm{~nm}$ continuous wave (cw) excitation is shown in Fig. 1(a) by the black dashed line. The PL signal shows a peak emission at $1375 \mathrm{~nm}$ and a full width at half maximum
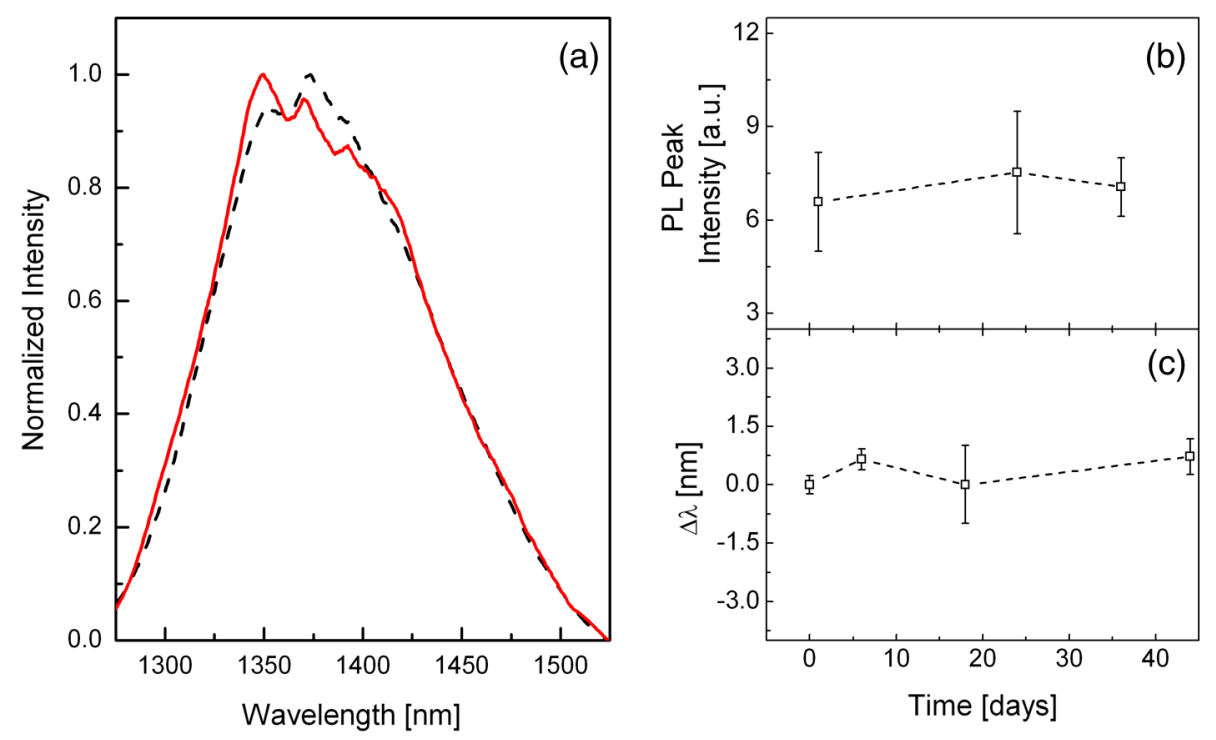

Fig. 1 (a) Normalized room temperature photoluminescence (PL) spectra of pure PbS nanocrystals (NCs) (black dashed line) and a Novolak-NC blend containing 4 vol\% of PbS drop cast onto an Si substrate (full red line). (b) PL peak intensity of a defined number of NCs embedded into Novolak polymer under nominally equal optical excitation as function of the time under ambient conditions after drop casting. (c) Shift of the emission peak wavelength of the compound in function of the time under ambient conditions after drop casting. 
(FWHM) of $\sim 120 \mathrm{~nm}$, which is due to the inhomogeneous size distribution of the NCs. However, these NCs, when kept in an ambient environment, tend to bleach and oxidize quickly, which causes the PL signal to vanish or severely blue-shift, respectively. ${ }^{10}$ The incorporation of the NCs into a host material is one way to counteract these effects. From a device point of view, where the stability of the optical properties of the NCs over time is of great importance, poly (methyl-methacrylate) (PMMA), which is commonly used in research as a host material for $\mathrm{NCs},{ }^{11,12}$ is not suitable because a large shift on the PL peak emission can still be observed over the course of several days. ${ }^{13}$ On the other hand, novel epoxy-based photoresists like SU-8 or mr-L 6005 XP have been shown to also keep their lithographic processability after the incorporation of NCs emitting in the visible wavelength regime. ${ }^{14,15}$ A compound material suitable for device applications at telecom wavelengths should consequently ensure high stability over a long period of time while still preserving the sensitivity to lithographic patterning.

In our case, the epoxy-based negative electron beam resist Novolak ARN 7700.18 from Allresist is used as a host material for the PbS NCs. Novolak-based polymer-NC blends containing different concentrations of NCs from 8 down to $0.1 \mathrm{vol} \%$ are mixed under nitrogen atmosphere to avoid any $\mathrm{NC}$ degradation throughout the compound fabrication process. In Fig. 1(a), the normalized PL spectrum of a polymer-NC blend containing $4 \mathrm{vol} \%$ of $\mathrm{PbS}$ drop cast onto a Si substrate is shown by the full red line. A perfect spectral agreement confront to the original pure $\mathrm{PbS}$ spectrum is found. The small intensity drops in both spectra of Fig. 1(a) at 1365 and $1385 \mathrm{~nm}$ correspond to atmospheric absorption.

The stability of intensity and spectral shape of the mixture over time is investigated and the results are presented in Figs. 1(b) and 1(c), respectively. In Fig. 1(b), the PL peak intensity of a fixed number of NCs embedded into Novolak polymer and coupled to a waveguide as discussed in a following paragraph is investigated over several weeks after drop casting. No degradation of the emission intensity is observed, showing that bleaching of the NCs in the polymer host is practically absent. Additionally, the spectral stability of the emission peak wavelength is shown in Fig. 1(c). As no spectral shift is observed after more than 40 days, we conclude that the NCs in the compound are also insensitive to oxidation. In between measurements, the samples were stored at ambient atmosphere.

Lithographic patterning imposes additional requirements on the compound, perhaps the most crucial one being the necessity to bake the material after exposure at $110^{\circ} \mathrm{C}$ for 2 min in order to crosslink the molecular chains of the negative resist so that the exposed areas are not removed during development. We thus examine the PL peak intensity of the polymer-NC blend while repeatedly heating the sample up to $115^{\circ} \mathrm{C}$ and cooling it back down to room temperature. Measurement results are presented in Fig. 2(a). By increasing the temperature of the sample, the observed PL peak intensity is lowered dramatically. This behavior can be a result of several effects discussed in the literature, such as the thermal activation between bright and dark excitonic states ${ }^{16}$ with subsequent nonradiative recombination or an increase of carrier-phonon interactions at elevated temperatures, ${ }^{17,18}$ although an exact mechanism is still under debate. ${ }^{19}$ By reducing again the temperature, however, the original PL peak intensity is restored. The heating and cooling steps were repeated several times on the same sample, thus confirming that the NCs do not experience temperatureinduced permanent degradation up to $115^{\circ} \mathrm{C}$.

Subsequently, the compound was spin coated on a photonic device and a $10 \times 10 \mu \mathrm{m}^{2}$ square was defined on top of a ring resonator using EBL. A scanning electron microscopy picture of the final device is shown in Fig. 2(c). Even though practically any kind of structure could be realized with this method, the rather large-scale patterning allows a straightforward comparison of the emission intensity in a confocal $\mu$-PL setup. The PL spectra of the polymer-NC layer before and after EBL are shown in Fig. 2(b). Neither a change of the PL intensity nor a shift of the emission peak wavelength is observed. Although the possibility for lithographic patterning without affecting the optical properties of the PbS NCs in the polymer is not essential for the work discussed in the following, this demonstrates the possibility to use this compound as an active material for on-chip applications and devices.

All this information indicates that the Novolak-based NC compound is a promising material for the fabrication of all-integrated hybrid photonic structures at telecom wavelengths, and in the rest of this article, it is used as the active medium. 

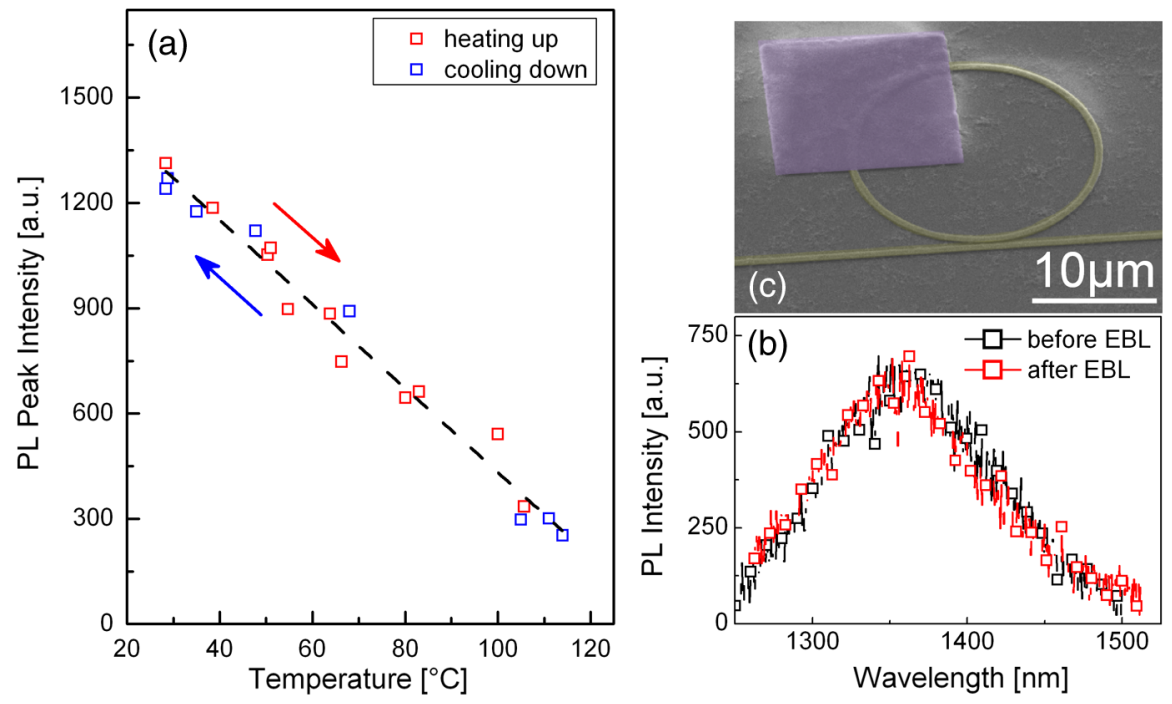

Fig. 2 (a) PL peak intensity of the Novolak-NC mixture at various temperatures during heating (red open squares) and cooling (blue open squares). (b) PL spectra of the Novolak-NC mixture before electron-beam lithography (EBL) (black line with open squares) and after EBL (red line with open squares). (c) SEM image of a $10 \times 10 \mu \mathrm{m}^{2}$ square of spin cast Novolak-NC mixture defined on $1 / 4$ of a photonic ring resonator by EBL.

\section{Device Design and Fabrication}

The first photonic devices for testing the integration of the PbS NCs are monomode waveguides coupled to ring resonators fabricated on a silicon on insulator wafer. All the processing of these samples was done by Amo Ltd. (Aachen, Germany) using EBL and inductively coupled plasma reactive ion etching (RIE). The 220-nm-high and 600-nm-wide Si waveguides are separated from the ring resonator (radius $r=10 \mu \mathrm{m}$ ) of the same cross-section by a gap of $150 \mathrm{~nm}$.

In order to limit the interaction between the polymer-NC compound and the waveguide structures to a specific region on the device, the samples are passivated by spin casting a layer of Microchem SU-8 2000.5 negative resist with a thickness of $500 \mathrm{~nm}$. The samples are then further processed using UV lithography in order to open $60 \times 30 \mu \mathrm{m}$ interaction windows around the ring resonators. As a final step of the sample preparation, the SU-8 is cured on a hotplate at $150^{\circ}$ $\mathrm{C}$ for $10 \mathrm{~min}$. These windows are then filled by drop casting $\sim 0.5 \mu \mathrm{l}$ of the polymer-NC mixture and subsequent curing of the device at $75^{\circ} \mathrm{C}$ for $1 \mathrm{~min}$. A scheme of the final device is shown in Fig. 3(b).

Due to the nearly equal refractive indices of SU-8 and the Novolak polymer (1.572 and 1.575 , respectively, at $1500 \mathrm{~nm}$ wavelength), ${ }^{20,21}$ no significant mode distortion is experienced by the light travelling in the waveguide across the interface between SU-8 and the Novolak-NC compound. Another advantage of using SU-8 as a passivation layer in combination with the Novolak-based compound is the fact that the hard-baked SU-8 is only removable using piranha wet etching or RIE, whereas the polymer-NC blend can easily be cleaned off the device using the Allresist AR 300-70 remover. This allows a quick and efficient swapping of mixtures of different $\mathrm{NC}$ concentrations without affecting the device itself and in particular the waveguide facets. Additionally, this method enables an investigation of the same device for different NC concentrations of the compound.

Figures 3(e) and 3(f) show full-vector three-dimensional (3-D) beam propagation method (BPM) simulations of the fundamental modes of the $\mathrm{Si}$ waveguide at $1.45 \mu \mathrm{m}$ wavelength for quasi transverse-electric (TE) and quasi transverse-magnetic (TM) polarization, respectively. The blue lines indicate the borders of the structures. In the TE case, most of the light is well confined inside the waveguide and the overlap factor $\Gamma$ (defined as the partial power of the guided light within the active material with respect to the total guided power) is calculated to be only 0.1 . Due to the waveguide cross-section dimensions, the TM mode is more weakly 
(a)

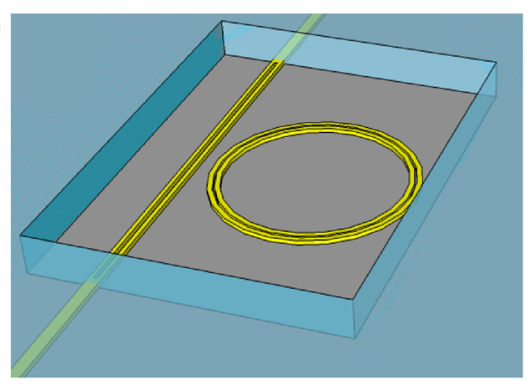

(b)

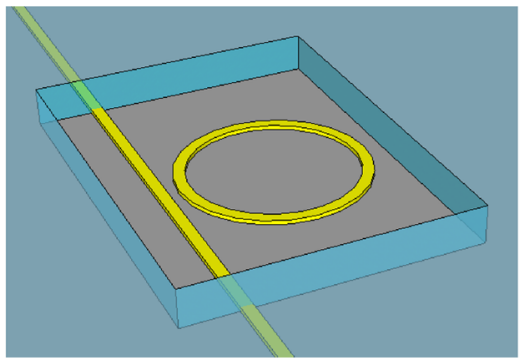

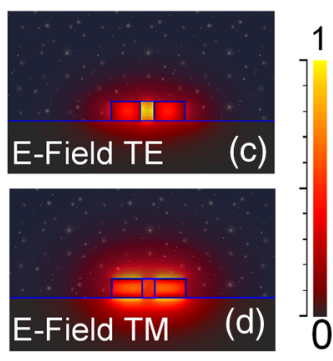

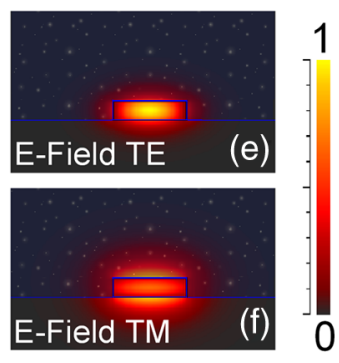

Fig. 3 Schematics of the vertically slotted (a) and unslotted (b) ring resonator-waveguide structure with the SU-8 interaction window. (c) to (e) show three-dimensional (3-D) beam propagation method (BPM) contour plots of the fundamental modes of the vertically slotted waveguide in TE (c) and TM (d) polarization and the unslotted waveguide in TE (e) and TM (f).

confined to the Si waveguide, and consequently $\Gamma=0.25$. The effective index of refraction $n_{\text {eff }}$ is calculated to be 2.63 and 2.04 for TE and TM, respectively.

In order to further increase the overlap factor and thus the interaction of the NCs with the mode of the waveguide, a vertically slotted structure ${ }^{22}$ was also designed. This high-index contrast photonic structure with deep-submicron feature size consists of a thin layer of low-index material sandwiched between two high-index walls. Compared to conventional waveguides, where the main part of the optical field is confined within the high-index core, a larger fraction of the guided mode is within the low-index region, which in our case is the polymer-NC compound. This effect occurs only for the polarization mode whose electric field is perpendicular to the slot interfaces, which in the case of the vertically slotted structures discussed here is TE.

The outer dimensions of the slot waveguides are similar to the ones already described above, but in addition, they feature 100-nm-wide vertical slots in the center. The gap between the (also slotted) ring resonator and the waveguide is $300 \mathrm{~nm}$. Figure 3(a) shows a sketch of such a vertically slotted device. Figure 3(c) represents 3-D BPM simulations of the fundamental mode of the Si slot waveguide for TE polarization. The field is affected by a discontinuity at the slot-wall interfaces, which increases the maxima of the optical field evanescent tails within the slot, and $\Gamma$ and $n_{\text {eff }}$ are calculated to be 0.38 and 2.03, respectively. A decomposition of the overlap factor $\Gamma=\Gamma_{\text {slot }}+\Gamma_{\text {out }}$ into contributions from inside $\left(\Gamma_{\text {slot }}\right)$ and outside $\left(\Gamma_{\text {out }}\right)$ the slot results in $\Gamma_{\text {slot }}=$ 0.15 and $\Gamma_{\text {out }}=0.23$. In TM polarization no such beneficial effect from the slot can be anticipated from the mode picture [Fig. 3(d)]. Although a slightly increased overlap factor $\Gamma$ of 0.4 due to weaker confinement to the waveguide and thus a smaller $n_{\text {eff }}=1.83$ are calculated, the value for $\Gamma_{\text {slot }}$ is only 0.06 .

\section{Transmission Measurements}

The transmission spectra of the devices for different NC concentrations were measured using a tunable infrared $\mathrm{cw}$ laser source fiber-coupled to a polarization-maintaining (PM) lensed fiber mounted on a piezo stage. The excitation power of the laser was set to $1 \mathrm{~mW}$ for all measurements. Light at the output of the waveguide was collected by a second PM fiber and coupled to an InGaAs detector. The input and collection fiber polarization were controlled by rotatable fiber holders. 


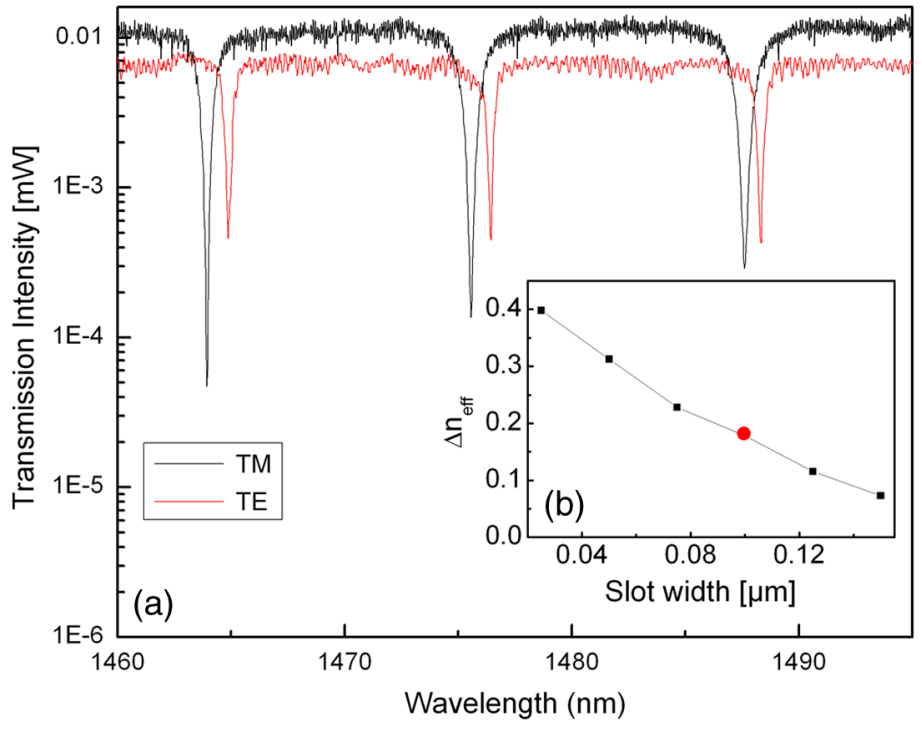

Fig. 4 (a) Transmission spectra of the vertically slotted ring resonators coupled to bus waveguides for an NC concentration of 2 vol\% in the Novolak matrix, where the red and black solid lines correspond to TE and TM polarization, respectively. The excitation power was set to $1 \mathrm{~mW}$. (b) 3-D BPM simulations of the difference in effective indices of refraction of the fundamental modes in TE and TM polarization $\Delta n_{\text {eff }}=n_{\text {eff,TE }}-n_{\text {eff,TM }}$ in function of the width of the vertical slot. The red dot indicates $\Delta n_{\text {eff }}$ for the structure investigated in this work.

Figure 4(a) shows transmission spectra of the vertically slotted device in TE (red solid line) and TM (black solid line) polarization for an NC concentration of 2 vol\%. Both the free spectral range (FSR) and the absolute spectral positions of the TE and TM resonances are very similar in the wavelength region investigated. For polarization-selective coupling of the $\mathrm{PbS}$ emission into the resonators, the spectral separation of TM and TE modes has to be controlled. One method to avoid the mode overlapping over a large spectral range is to increase the difference of the effective indices of refraction between TE and TM polarization $\Delta n_{\text {eff }}=n_{\text {eff,TE }}-n_{\text {eff,TM }}$, resulting in a relative shift between the polarization-specific FSRs. For the vertically slotted structures, decreasing the width of the slot from 100 to $50 \mathrm{~nm}$ would cause an increase of $\Delta n_{\text {eff }}$ from 0.175 to 0.315 , as shown by the results of 3-D BPM simulations in Fig. 4(b). Thus, by a proper choice of the slot width, overlapping TE and TM resonances can be avoided for any spectral region of interest.

The total quality factors $Q_{t}$ (averaged over five central resonances) are $\sim 4200$ and $\sim 1400$ in $\mathrm{TE}$ and TM polarization, respectively. $\mathrm{Q}_{t}$ denotes the as-measured $\mathrm{Q}$ factor of a resonator coupled to a waveguide. Depending on the gap separation between the bus-waveguide and the ring resonator, the coupling efficiency between them changes, which consequently modifies the associated loss channel seen by the resonator. Although this is generally inevitable for coupled structures as discussed in this work, we note that the waveguide-resonator separation can be tuned toward strong undercoupling in order to approach the intrinsic $Q$ factor $Q_{i}$ of only the ring resonator, which is related to the losses caused by scattering, absorption, and radiative processes. ${ }^{23}$ Following Ref. 9, the $\mathrm{Q}_{\mathrm{i}}$ values of the different photonic structures are extracted from the obtained transmission measurements and represented in Fig. 5(a) for different NC concentrations.

By increasing the NC concentration in the active NC-polymer compound, it is clear that depending on the resonator type investigated, and above a certain concentration scattering, losses induced by the NCs coupled to the resonators start to degrade their Q factor. In the case of the nonslotted resonators, the effect of the NCs on the losses starts to appear between 4 and 6 vol\%. On the other hand, as the slotted resonators have larger overlaps with the polymer-NC compound, the NC-induced scattering losses degrade the obtained $\mathrm{Q}$ values already beyond a concentration of $2 \mathrm{vol} \%$. Below these critical concentrations, the resonators show similar Q factors as unloaded ones, which are covered only by pure Novolak polymer without any NCs. 

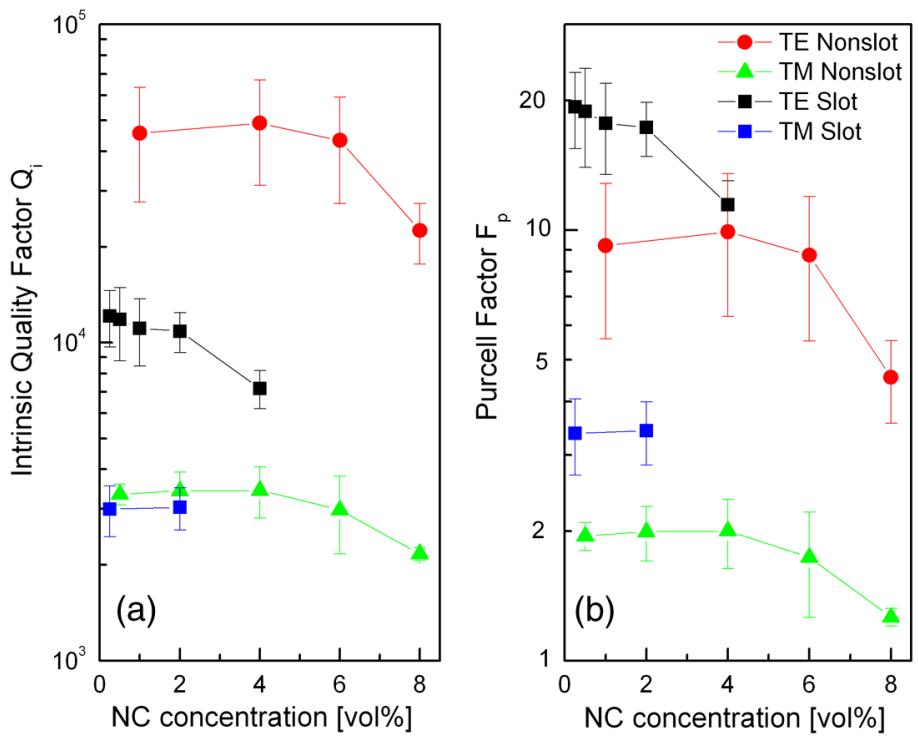

Fig. 5 Intrinsic quality $(Q)$ factors $Q_{i}(a)$ calculated from the measured transmission spectra and calculated Purcell factors $F_{p}$ (b) as function of the NC concentrations of slotted ring resonator structures in TE (black squares) and TM (blue squares) polarization and unslotted devices in TE (red circles) and TM (green triangles).

The replacement of the polymer-NC compound was repeated several times for different NC concentrations in order to verify that no residual NCs remain on the devices after the cleaning step.

\section{Photoluminescence Measurements and Purcell Factor}

The intrinsic Q factor is highest for nonslotted ring resonators for TE polarization, where the guided light experiences the highest confinement to the Si. However, more important than the Q factor for emitters coupled to resonators is the Purcell factor, which describes the enhancement of the spontaneous emission rate of an emitter into a resonator mode with respect to its integrated emission rate into all vacuum modes. ${ }^{24}$ According to Ref. 25, the Purcell factor of an optical resonator is defined as $\mathrm{F}_{p}=\Gamma /\left(4 \pi^{2}\right)\left(\lambda / n_{\text {eff }}\right)^{3}\left(\mathrm{Q}_{\mathrm{i}} / V\right)$, where $V$ denotes the mode volume calculated using 3-D finite-difference time-domain simulations. For the nonslotted device, mode volumes of resonances around $1.45 \mu \mathrm{m}$ wavelength in TE and TM are calculated to be 2.1 and $3.9 \mu \mathrm{m}^{3}$, respectively. For the slotted ring resonators, mode volumes in TE and TM of 2.2 and $4.4 \mu \mathrm{m}^{3}$ are calculated. Figure 5(b) shows the calculated Purcell factors of the different devices for varying $\mathrm{NC}$ concentrations, where the highest $\mathrm{F}_{p}$ of 20 is obtained for the slotted device in TE polarization, a factor of 2 higher than $\mathrm{F}_{p}$ for the unslotted device in TE, which is $\sim 10$. This demonstrates that the slotted structure is more suitable as an active photonic building block with NCs acting as emitters.

It should be noted that the Purcell factor, which is by definition a property of a cavity independent of emitters coupled to it, only truly holds for perfect emitters with negligible homogeneous linewidth broadening and without any nonradiative decay channel. However, even though colloidal $\mathrm{PbS} \mathrm{NCs}$ at room temperature are not considered to be perfect emitters, the improvement of $\mathrm{F}_{p}$ from introducing a vertical slot into the structure holds also for nonideal emitters. Consequently, PL measurements were performed on the slotted device.

For the experimental investigation of the PL performance of the slotted device for different $\mathrm{NC}$ concentrations, an AlGaInP $\mathrm{cw}$ laser with an emission wavelength of $657 \mathrm{~nm}$ was focused through a microscope objective onto the ring resonator, with a spot size only slightly larger than the diameter of the ring $(\sim 20 \mu \mathrm{m})$. The excitation intensity on the sample was kept constant at $1.64 \mathrm{~kW} / \mathrm{cm}^{2}$ for all measurements. The PL signal is collected at one output facet of the waveguide through a PM lensed fiber and a fiber-coupled polarizer, after which it is dispersed by a fiber-coupled monochromator connected to an InGaAs photodiode array detector (nominal 

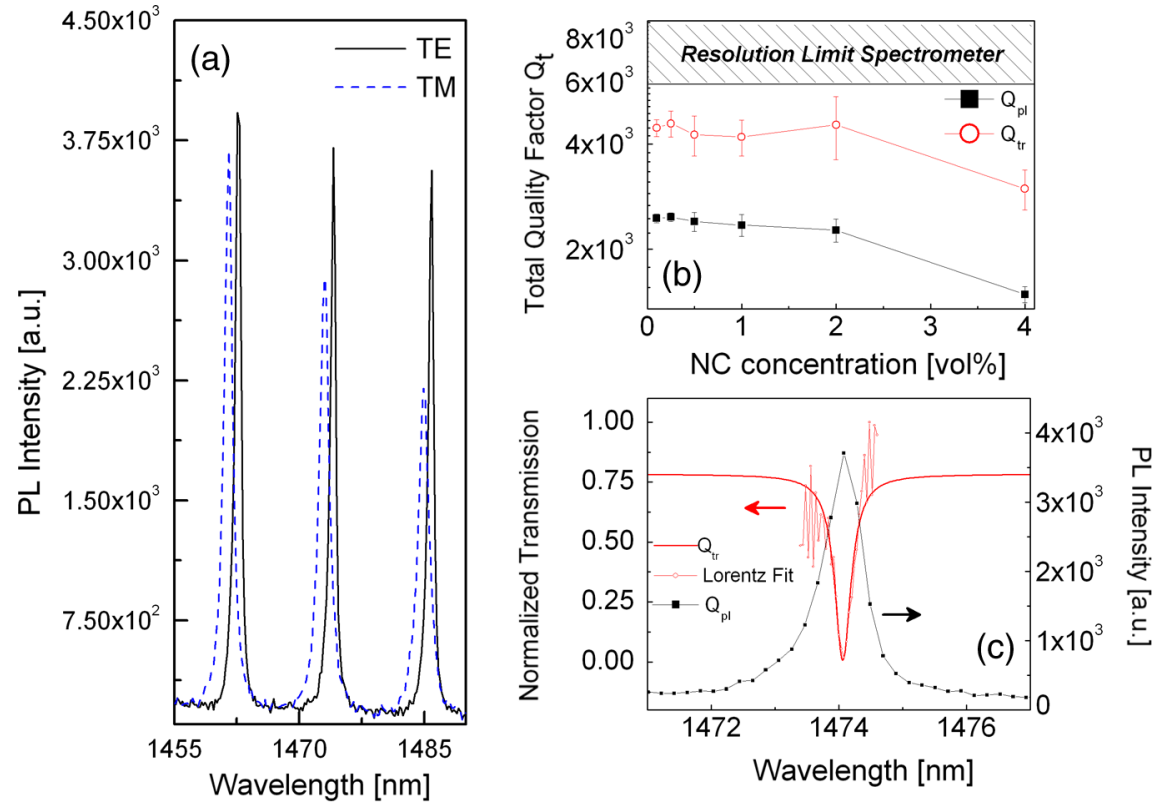

Fig. 6 (a) Room temperature PL spectrum of the vertically slotted ring resonator device for an NC concentration of 2 vol\% in the Novolak host with a pump excitation intensity of $1.64 \mathrm{~kW} / \mathrm{cm}^{2}$ measured at one output facet of the waveguide in TE (black solid line) and TM (blue dashed line). (b) Total $Q$ factor $Q_{t}$ directly obtained from transmission (black squares, $Q_{t r}$ ) and $P L$ measurements (red open circles, $Q_{p l}$ ) of the vertically slotted device in TE polarization for different NC concentrations. The dashed area indicates the limit of our PL measurement setup expressed in terms of $Q$ factors. (c) Normalized transmission spectrum (red squares $Q_{\mathrm{tr}}$, solid red line corresponds to a Lorentzian fit) and PL spectrum (black squares, $\mathrm{Q}_{\mathrm{pl}}$ ) of the same cavity resonance of the slotted ring resonator structure in TE polarization for an NC concentration of $2 \mathrm{vol} \%$.

resolution $0.2 \mathrm{~nm}$ ). In Fig. 6(a), the PL spectra of the device covered with a polymer-NC compound containing 2 vol\% of $\mathrm{PbS}$ are plotted in TE (black solid line) and TM polarization (blue dashed line), demonstrating that the PL signal can be investigated in one polarization while the other polarization is completely canceled in the spectrum.

Figure 6(b) shows a comparison of $\mathrm{Q}_{\mathrm{t}}$ (averaged over five central resonances) obtained from transmission and PL measurements in TE polarization. Even though both curves still show the effect of NC-induced scattering losses at concentrations $>2 \mathrm{vol} \%$, an approximately two times smaller $\mathrm{Q}_{t}$ is observed in PL experiments as compared to transmission experiments. The resolution limit of the monochromator used for PL measurements expressed as Q factor is also indicated in Fig. 6(b). It was experimentally determined by measuring the FWHM of the tunable $\mathrm{cw}$ laser source (used for the transmission measurements), which was injected into the photonic device detuned from the ring resonances and spectrally resolved by the monochromator. Evidently, the resolution limit of the PL measurement setup does not limit the $\mathrm{Q}_{t}$ values measured in PL experiments.

In Fig. 6(c), a spectrum of the same TE resonance is plotted from transmission and PL measurements. Since no shift in terms of resonance wavelength is observed, we assume that thermal effects are negligible, even more so because a polymer top cladding is known to reduce the resonance wavelength temperature dependence of Si microring resonators. ${ }^{26}$ Transmission measurements were also performed while the PL top excitation laser was illuminating the resonator in order to rule out any effects of the excitation laser on the different $Q_{t}$ factors observed in transmission and PL experiments. We tentatively assign this difference in $\mathrm{Q}_{t}$ factors to the NC homogeneous emission linewidth being not significantly smaller than that of the cavity, thereby imposing an upper limit for the PL Q factors and the utilizable Purcell enhancement for these NCs. ${ }^{27}$ To estimate the homogeneous linewidth of PbS NCs emitting in the telecom wavelength regime and the magnitude of the Purcell factor in our ring resonators, time-resolved PL experiments will be performed. 


\section{Conclusion}

In conclusion, we have demonstrated the functionalization of an epoxy-based negative photoresist with colloidal $\mathrm{PbS} \mathrm{NCs}$ emitting at telecom wavelengths. We have investigated the intensity and spectral stability of this compound, which shows unaltered optical properties weeks after preparation. No permanent temperature-induced degradation of the PL signal is observed up to $115^{\circ} \mathrm{C}$, thus enabling lithographic patterning of the polymer-NC mixture for on-chip applications. Our choice of materials allows for one and the same all-integrated Si-based ring resonator to investigate its performance as a function of the $\mathrm{NC}$ concentration in the polymer, which is coupled to the resonators via drop casting. A critical concentration beyond which NC-induced scattering losses start to degrade the $Q$ factors of the devices was determined. Solid and vertically slotted cavities were compared in terms of Q factors and ideal Purcell factors. PL experiments on the vertically slotted resonators show high-intensity peaks at the facets of the output waveguide. Further investigations are needed to determine the origin of the different $\mathrm{Q}$ factors observed in the transmission and PL experiments for the slotted ring resonators.

Finally, the method presented in this work can also be applied for different NCs, enabling simple tuning of the working bandwidth of NC-based optoelectronic chips within the transparency region of $\mathrm{Si}$ photonics.

\section{Acknowledgments}

This work was supported by the Austrian Nanoinitiative within the PLATON project cluster under project numbers $819654,819655,819656,834913$, and 834924 as well as the Austrian Science Foundation FWF-F2521 (SFB025 IR-ON). We also acknowledge AMO GmbH in Aachen (Germany) for their support and the fabrication of the SOI waveguides and resonators.

\section{References}

1. B. Jalali and S. Fathpour, "Silicon photonics," J. Lightwave Technol. 24(12), 4600-4615 (2006), http://dx.doi.org/10.1109/JLT.2006.885782.

2. D. V. Talapin et al., "Prospects of colloidal nanocrystals for electronic and optoelectronic applications," Chem. Rev. 110(1), 389-458 (2010), http://dx.doi.org/10.1021/cr900137k.

3. P. Guyot-Sionnest, "Electrical transport in colloidal quantum dot films," J. Phys. Chem. Lett. 3(9), 1169-1175 (2012), http://dx.doi.org/10.1021/jz300048y.

4. A. Omari et al., "Role of interband and photoinduced absorption in the nonlinear reflection and absorption of resonantly excited $\mathrm{PbS}$ quantum dots around $1550 \mathrm{~nm}$," Phys. Rev. B. 85(11), 115318 (2012), http://dx.doi.org/10.1103/PhysRevB.85.115318.

5. M. T. Rakher et al., "Spectroscopy of $1.55 \mu \mathrm{m} \mathrm{PbS}$ quantum dots on Si photonic crystal cavities with a fiber taper waveguide," Appl. Phys. Lett. 96(16), 161108 (2010), http://dx.doi .org/10.1063/1.3396988.

6. J. Heo et al., "Electroluminescence from silicon-based photonic crystal microcavities with PbSe quantum dots," Opt. Lett. 35(4), 547-549 (2010), http://dx.doi.org/10.1364/OL.35 .000547 .

7. Y. Olsson et al., "Fabrication and optical properties of polymeric waveguides containing nanocrystalline quantum dots," Appl. Phys. Lett. 85(19), 4469-4471 (2004), http://dx .doi.org/10.1063/1.1818723.

8. N. Tessler et al., "Efficient near-infrared polymer nanocrystal light-emitting diodes," Science 295(5559), 1506-1508 (2002), http://dx.doi.org/10.1126/science.1068153.

9. M. Humer et al., "Integration, photostability and spontaneous emission rate enhancement of colloidal $\mathrm{PbS}$ nanocrystals for Si-based photonics at telecom wavelengths," Opt. Express 21(16), 18680-18688 (2013), http://dx.doi.org/10.1364/OE.21.018680.

10. J. J. Peterson and T. D. Krauss, "Fluorescence spectroscopy of single lead sulfide quantum dots," Nano Lett. 6(3), 510-514 (2006), http://dx.doi.org/10.1021/n10525756.

11. D. F. Dorfner et al., "Optical characterization of silicon on insulator photonic crystal nanocavities infiltrated with colloidal PbS quantum dots," Appl. Phys. Lett. 91(23), 23311 (2007), http://dx.doi.org/10.1063/1.2822441. 
12. R. Bose et al., "Weak coupling interactions of colloidal lead sulfide nanocrystals with silicon photonic crystal nanocavities near $1.55 \mu \mathrm{m}$ at room temperature," Appl. Phys. Lett. 90(11), 111117 (2007), http://dx.doi.org/10.1063/1.2714097.

13. D. W. Grund, B. Olbricht, and D. W. Prather, "Quantum dot composite light sources," Proc. SPIE 7953, 795302 (2011), http://dx.doi.org/10.1117/12.875227.

14. C. Ingrosso et al., "An epoxy photoresist modified by luminescent nanocrystals for the fabrication of 3D high-aspect-ratio microstructures," Adv. Funct. Mater. 17(13), 20092017 (2007), http://dx.doi.org/10.1002/(ISSN)1616-3028.

15. A. Qualtieri et al., "Advances in resist materials and processing technology: photonic devices fabricated by direct lithography of resist/colloidal nanocrystals blend," Chapter 7 in Lithography, M. Wang, Ed., pp. 115-130, InTech Publishing, Rijeka, Croatia (2010).

16. V. I. Klimov, "Mechanisms for photogeneration and recombination of multiexcitons in semiconductor nanocrystals: implications for lasing and solar energy conversion," J. Phys. Chem. B 110(34), 16827-16845 (2006), http://dx.doi.org/10.1021/jp0615959.

17. K. Szendrei et al., "Exploring the origin of the temperature-dependent behavior of $\mathrm{PbS}$ nanocrystal thin films and solar cells," Adv. Funct. Mater. 22(8), 1598-1605 (2012), http://dx.doi.org/10.1002/adfm.v22.8.

18. L. Turyanska et al., "Temperature dependence of the photoluminescence emission from thiol-capped PbS quantum dots," Appl. Phys. Lett. 90(10), 101913 (2007), http://dx.doi .org/10.1063/1.2711529.

19. B. Ullrich, J. S. Wang, and G. J. Brown, "Comment on 'temperature dependent optical properties of PbS nanocrystals'," Nanotechnology 24(28), 288001 (2013), http://dx.doi .org/10.1088/0957-4484/24/28/288001.

20. W. H. Wong, J. Zhou, and E. Y. B. Pun, "Low-loss polymeric waveguides using electronbeam direct writing," Appl. Phys. Lett. 78(15), 2110-2112 (2001), http://dx.doi.org/10 $.1063 / 1.1361287$.

21. C. S. Huang and W. C. Wang, "Large-core single-mode SU8 rib waveguide using solventassisted microcontact molding," Appl. Opt. 47(25), 4540-4547 (2008), http://dx.doi.org/10 .1364/AO.47.004540.

22. V. R. Almeida et al., "Guiding and confining light in void nanostructure," Opt. Lett. 29(11), 1209-1211 (2004), http://dx.doi.org/10.1364/OL.29.001209.

23. R. Guider et al., "NanoSi low loss horizontal slot waveguides coupled to high Q ring resonators," Opt. Express 17(23), 20762-20770 (2009), http://dx.doi.org/10.1364/OE.17 .020762 .

24. E. M. Purcell, "Spontaneous emission probabilities at radio frequencies," Phys. Rev. 69, 681 (1946), http://dx.doi.org/10.1103/PhysRev.69.37.

25. T. Baba and D. Sano, "Low-threshold lasing and Purcell effect in microdisk lasers at room temperature," IEEE J. Sel. Topics Quantum Electron. 9(5), 1340-1346 (2003), http://dx.doi .org/10.1109/JSTQE.2003.819464.

26. J. Teng et al., "Athermal silicon-on-insulator ring resonators by overlaying a polymer cladding on narrowed waveguides," Opt. Express 17(17), 14627-14633 (2009), http://dx.doi .org/10.1364/OE.17.014627.

27. A. Pitanti et al., "Probing the spontaneous emission dynamics in Si-nanocrystals-based microdisk resonators" Phys. Rev. Lett. 104(10), 103901 (2010), http://dx.doi.org/10 .1103/PhysRevLett.104.103901.

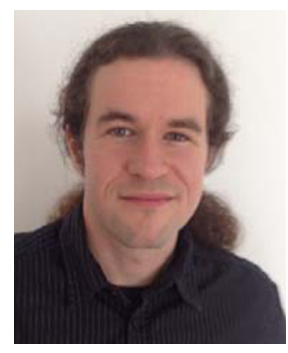

Markus Humer is a graduate student at the Johannes Kepler University (JKU) of Linz. He received his diploma in technical physics in 2008 in the nano-MIR research group of Professor Dr. Wolfgang Heiß at the Institute of Solid-State Physics at JKU. He is currently working toward his $\mathrm{PhD}$ degree in the Nanostructures4Optoelectronics research group of Dr. Thomas Fromherz at the Institute of Semiconductor Physics. His current research interests include colloidal nanocrystal-based photonic devices, nanoplasmonics, cavity quantum electrodynamics, slow light, and graphene. 


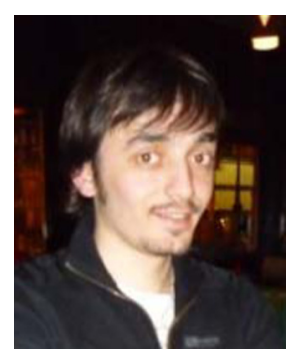

Romain Guider received his degree in optoelectronics engineering from the École Nationale Supérieure de Sciences Appliquées et de Technologie, Lannion, France, in 2006, and his PhD degree from the University of Trento, Trento, Italy, in 2009. His doctoral research focused on the study of new nanosilicon waveguides and nanophotonics structures, more particularly on slot waveguides, ring and disk resonators. In January 2011, he joined the Institut für Halbleiter und Festkörperphysik, Linz University, Austria, as a postdoctoral fellow. His research interests include design, fabrication, and characterization of new Si-based photonic structures for on-chip applications.

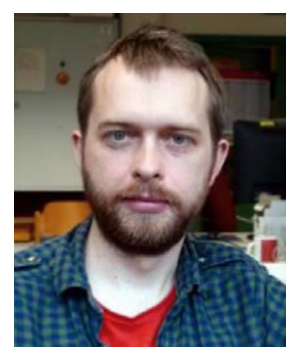

Florian Hackl is a graduate student at the JKU of Linz and received his diploma in technical physics in 2012 in the research group of Professor Dr. Günther Bauer at the Institute of Semiconductor Physics at the JKU. $\mathrm{He}$ is currently working on his $\mathrm{PhD}$ within the Infra Red Optical Nanostructures project in the research group of Dr. Thomas Fromherz at the Institute of Semiconductor Physics. His current research interests include Si-based optical nanostructures, $\mu$-photoluminescence, and timeresolved spectroscopy in the telecom spectral range.

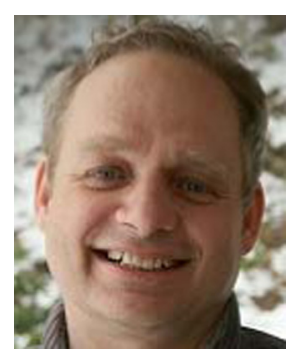

Thomas Fromherz received his $\mathrm{PhD}$ degree in technical sciences from JKU, Linz, Austria, in 1994. He was an Erwin Schrödinger fellow at the Quantum Institute of the University of California, Santa Barbara, in 1996 and worked as a research engineer in the Si Foundry Austria Microsystems International AG, Styria, Austria, from 1997 to 1998, and in the organic solar cell start-up company Quantum Solar Energy Linz, Austria, from 1998 to 2000. In 2000, he rejoined JKU as a research assistant at the Institute of Semiconductor and Solid State Physics and became an assistant professor in 2006. His current research interests include Si-based optoelectronics for the infrared and telecom spectral region. 